OPEN ACCESS

Edited by:

Alessandro Isidori,

AORMN Hospital, Italy

Reviewed by:

Claudio Cerchione,

Romagnolo Scientific Institute for the

Study and Treatment of Tumors

(IRCCS), Italy

Valentino Conter,

Fondazione MBBM, Italy

${ }^{*}$ Correspondence:

Benoit Brethon

benoit.brethon@aphp.fr

${ }^{+}$These authors have contributed equally to this work

Specialty section:

This article was submitted to

Hematologic Malignancies,

a section of the journal

Frontiers in Oncology

Received: 04 December 2020

Accepted: 20 January 2021

Published: 26 February 2021

Citation:

Brethon B, Lainey E, Caye-Eude A, Grain A, Fenneteau O, Yakouben $K$, Roupret-Serzec J, Le Mouel L, Cavé H and Baruchel A (2021) Case Report: Targeting 2 Antigens as a Promising Strategy in Mixed Phenotype Acute Leukemia: Combination of

Blinatumomab With Gemtuzumab Ozogamicin in an Infant With a KMT2A-Rearranged Leukemia.

Front. Oncol. 11:637951.

doi: 10.3389/fonc.2021.637951

\section{Case Report: Targeting 2 Antigens as a Promising Strategy in Mixed Phenotype Acute Leukemia: Combination of Blinatumomab With Gemtuzumab Ozogamicin in an Infant With a KMT2A-Rearranged Leukemia}

\author{
Benoît Brethon ${ }^{1 *}$, Elodie Lainey ${ }^{2,3 \dagger}$, Aurélie Caye-Eude ${ }^{3,4 \dagger}$, Audrey Grain ${ }^{1}$, \\ Odile Fenneteau ${ }^{2}$, Karima Yakouben ${ }^{1}$, Julie Roupret-Serzec ${ }^{1,5}$, Lou Le Mouel ${ }^{1}$, \\ Hélène Cavé ${ }^{3,4}$ and André Baruchel ${ }^{1,6}$

\begin{abstract}
${ }^{1}$ Department of Pediatric Hematology, University Robert Debre Hospital, Assistance Publique Hôpitaux de Paris (APHP), Hospital, APHP, Paris, France, ${ }^{6}$ University Institute of Hematology, University Saint-Louis Hospital, APHP, Paris, France
\end{abstract} \\ Paris, France, ${ }^{2}$ Department of Biological Hematology, University Robert Debre Hospital, APHP, Paris, France, ${ }^{3}$ INSERM \\ UMR_S1131, Institut de Recherche Saint-Louis, University of Paris, Paris, France, ${ }^{4}$ Department of Genetics, University \\ Robert Debre Hospital, APHP, Paris, France, ${ }^{5}$ Transversal Unit for Therapeutic Patient Education, University Robert Debre
}

Mixed phenotype acute leukemia (MPAL) accounts for 2-5\% of leukemia in children. MPAL are at higher risk of induction failure. Lineage switch ( $B$ to $M$ or vice versa) or persistence of only the lymphoid or myeloid clone is frequently observed in biphenotypic/ bilineal cases, highlighting their lineage plasticity. The prognosis of MPAL remains bleak, with an event-free survival (EFS) of less than $50 \%$ in children. A lymphoid-type therapeutic approach appears to be more effective but failures to achieve complete remission (CR) remain significant. KMT2A fusions account for $75-80 \%$ of leukemia in infants under one year of age and remains a major pejorative prognostic factor in the Interfant-06 protocol with a 6 years EFS of only $36 \%$. The search for other therapeutic approaches, in particular immunotherapies that are able to eradicate all MPAL clones, is a major issue. We describe here the feasibility and tolerance of the combination of two targeted immunotherapies, blinatumomab and Gemtuzumab Ozogamicin, in a 4-year-old infant with a primary refractory KTM2A-rearranged MPAL. Our main concern was to determine how to associate these two immunotherapies and we describe how we decided to do it with the parents' agreement. The good MRD response on the two clones made it possible to continue the curative intent with a hematopoietic stem cell transplant at 9 months of age. Despite a relapse at M11 post-transplant because of the recurrence of a pro-B clone retaining the initial lymphoid phenotype, the child is now 36 months old, in persistent 
negative MRD CR2 for 12 months after a salvage chemotherapy and an autologous CAR T cells infusion, with no known sequelae to date. This case study can thus lead to the idea of a sequential combination of two immunotherapies targeting two distinct leukemic subclones (or even a single biphenotypic clone), as a potential one to be tested prospectively in children MPAL and even possibly all KMT2A-rearranged infant ALL.

Keywords: mixed phenotype acute leukemia, infant, children, blinatumomab, gemtuzumab ozogamicin

\section{INTRODUCTION}

Mixed phenotype acute leukemia (MPAL) accounts for $2 \%-5 \%$ of leukemia in children (1). It is a heterogeneous group of diseases whose immunophenotypic definition has changed over time (2-4). The current WHO classification recognizes two subclassifications of MPAL (biphenotypic and bilineal) with mostly similar clinical and genetics characteristics. MPAL are at higher risk of induction failure. Lineage switch (B to $M$ or vice versa) or persistence of only the lymphoid or myeloid clone is frequently observed in biphenotypic/bilineal cases, highlighting their lineage plasticity. Prognosis of MPAL remains bleak, with an event-free survival (EFS) of less than $50 \%$ in children $(1,5-7)$. A lymphoid-type therapeutic approach appears to be more effective $(6,7)$ but failures to achieve complete remission (CR) remain significant. KMT2A fusions account for $75 \%-80 \%$ of leukemia in infants less than one year of age with typically an early pro-B CD10neg phenotype. Many cases express myeloid antigens (e.g., CD15/65, CD33, or weak MPO) but should not be considered MPAL unless unequivocal evidence of monoblastic differentiation ( $\geq 20 \%$ of blasts) or bright MPO expression, $<5 \%$ of all cases; KTM2A rearrangement remains a major pejorative prognostic factor in the international Interfant- 06 protocol and confers poor prognosis. The 6 years EFS for the low risk group (KMT2A germline patients) was $73.9 \%$ versus $36 \%$ for the KMT2A-rearranged group (8). The search for other therapeutic approaches, in particular immunotherapies that are able to eradicate all MPAL clones, is a major issue (9). We describe here the feasibility of the combination of two targeted immunotherapies in an infant with a primary refractory KTM2A-rearranged MPAL.

\section{CASE REPORT}

Four-month-old little girl presented in February 2018 with asthenia, pallor, cutaneous hemorrhages, hepato-splenomegaly, and superior airways obstructive respiratory signs. Maximum white blood cells count was 762 G/L. Workup showed a spontaneous tumor lysis syndrome without renal failure with a grade 3 biological disseminated intravascular coagulation. HCoV-NL63 (non-SARS) coronavirus was found in the

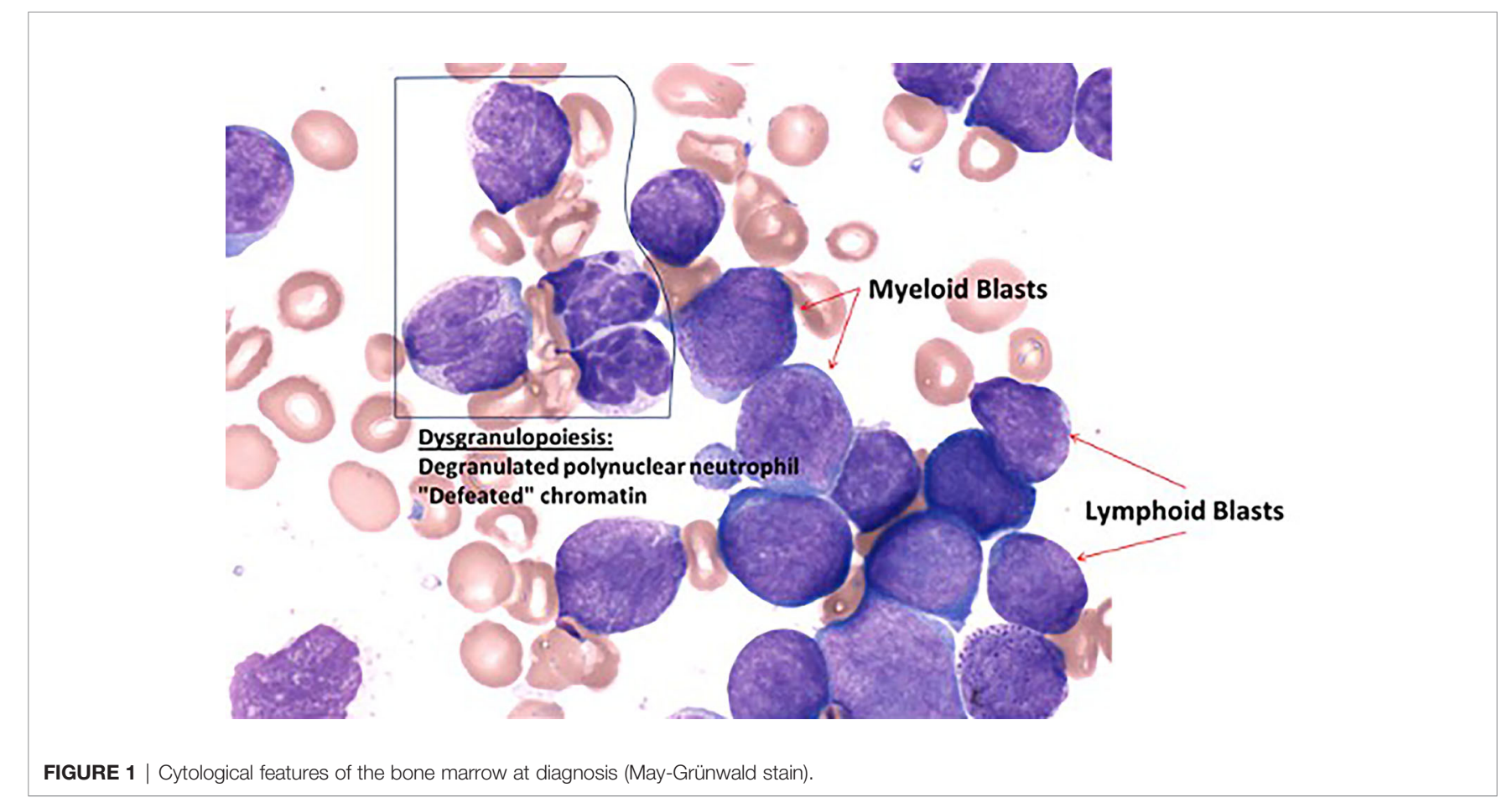




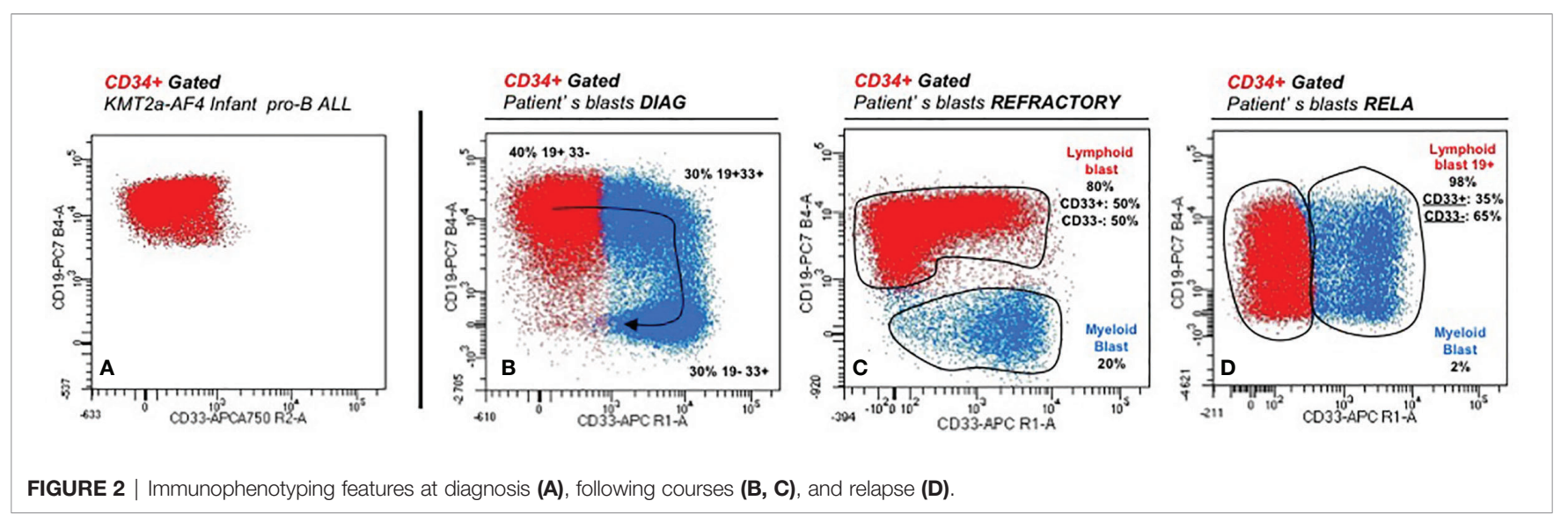

nasopharyngeal swab. Bone marrow was rich, infiltrated by $80 \%$ of lymphoblasts and $9 \%$ of resembling myeloblasts with fine granulations but peroxidase-negative. Atypical dysgranulopoiesis was also noted (Figure 1). Flow cytometry (FCM) showed two distinct CD34+ CD135+ (FLT3-R) populations evoking a MPAL (B/myeloid) diagnosis according to the WHO 2016. Pro-B clone (70\% of blasts) was CD19bright, CD22dim partial, cCD79a partial, CD20-, CD24dim without surface Kappa/Lambda or cytoplasmic mu-chain and coexpressed on some blasts (30\% of CD19+) several myeloid antigens (CD11c/CD64/CD15/CD33). Monocytic clone (30\%) was CD19-, cCD79a-, MPO-, CD33/11b/65/15/11c/64bright with partial CD14 (15\%) and partial co-expression of lymphoid markers CD22 and CD24 showing a continuum of expression/differentiation between the "pure" lymphoid and myeloid clones (Figure 2A). FISH evidenced KTM2A rearrangement in $93 \%$ of the nuclei. The presence of a KMT2A-AFF1 (also known as MLL-AF4) fusion was confirmed using RT-PCR. A missense mutation of the FLT3 gene $(43 \%$ allelic frequency) was found (Figure 3A). No deletion or duplication was found using MLPA (MRC HOLLAND kit P335-C1). Cerebrospinal fluid status was CNS3.

The child was treated according to the recommendations of the high-risk arm of the Interfant-06 protocol. A poor prednisone response was observed at D8 (blasts $127.9 \mathrm{G} / \mathrm{L}$ ). The CSF was normalized after three intrathecal injections. Unfortunately, CR was not achieved at the end of induction with a M2 bone marrow ( $9 \%$ blasts), progressing to a M3 bone marrow ( $32 \%$ blasts) 15 days after beginning the IB consolidation phase (cyclophosphamide/cytarabine/6mercaptopurine). A course of high-dose cytarabine-based chemotherapy $\left(2.25 \mathrm{~g} / \mathrm{m}^{2}\right.$ bid for 3 days) with amsidine (75 $\mathrm{mg} / \mathrm{m}^{2}$ per day for 3 days) failed with FCM showing the persistence of both B-lymphoid (80\%) and myeloid (20\%) blasts (Figure 2B).

After having obtained the informed consent of the parents and the authorization of the French national drug safety agency (ANSM), multidisciplinary team decision was to attack specifically each of the two phenotypically distinct leukemic clones with a combination of Blinatumomab (BLIN) and
A

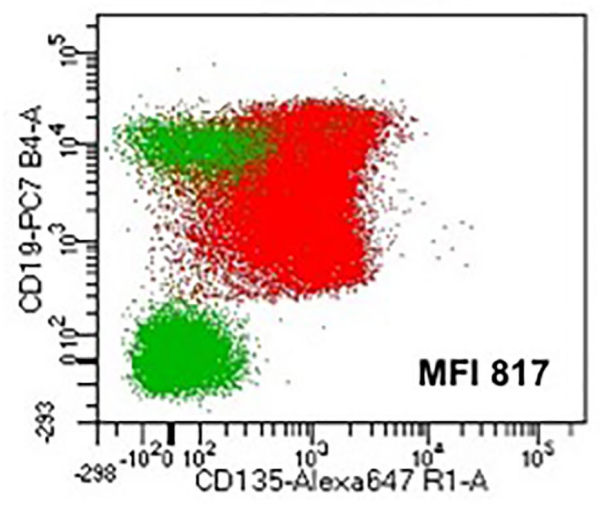

B

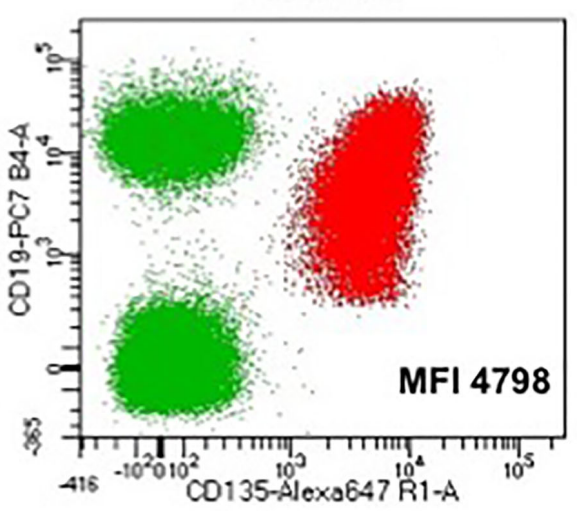

FIGURE 3 | Expression of the FLT3 receptor (CD135) on the blast population at diagnosis and at relapse showing a six times more expression at relapse (B) than at diagnosis (A) (MFI, mean fluorescence intensity). 
Gemtuzumab Ozogamicin (GO). BLIN was introduced first in May 2018 at a dose of $5 \mathrm{microg} / \mathrm{m}^{2} /$ day for 7 days and then increased at $15 \mathrm{microg} / \mathrm{m}^{2} /$ day. $\mathrm{GO}\left(3 \mathrm{mg} / \mathrm{m}^{2}\right)$ was added at D11, D15, D18 with triple intrathecal injections every 15 days (methotrexate, cytarabine, methylprednisolone). At D25, generalized seizures appeared in a febrile context, lasting for 5 min to stop BLIN. All radiological, biological, and microbiological documentations were negative. BLIN was resumed at D30 and continued until D73. One additional dose of GO $3 \mathrm{mg} / \mathrm{m}^{2}$ was administered at D44 for consolidation. The only other grade $3 / 4$ toxicity was an undocumented febrile neutropenia; no liver toxicity including sinusoidal obstructive syndrome (SOS) was seen. In terms of response, CR1 was obtained at D38 BLIN/GO. Minimal residual disease (MRD) as undetectable at the threshold of $10^{-4}$ by FCM and $10^{-5}$ using genomic PCR established on the KTM2A breakpoint. This was confirmed at the time of discontinuation of BLIN (administered for 68 days in total). At that time, there were still circulating Tlymphocytes $\left(\mathrm{CD} 3+780 / \mathrm{mm}^{3}, \mathrm{CD} 4+\right.$ at $\left.603 / \mathrm{mm}^{3}\right)$ but no Blymphocytes were seen as expected. This good response made it possible to continue the curative intent with a hematopoietic stem cell transplant in August 2018, i.e., at 9 months of age, after a conditioning regimen combining busulfan, fludarabine, and thiotepa. SOS prophylaxis consisted in defibrotide. A full donor chimerism was seen at mol and mo2. There was no acute GVHD or hepatic SOS. Ciclosporin A was stopped at mo3. MRD was negative again at M6 with both technics.

Unfortunately, a combined relapse occurred 11 months after HCST (child aged 21 months), with invasion of sinuses and orbits, a CNS2 CSF with 54\% of lymphoid blasts in the bone marrow. FCM confirmed the recurrence of a pro-B clone retaining initial phenotype (CD19bright, CD10-, partial CD33 but no monocytic antigens), with FLT3-R overexpression as in diagnosis (Figure 2C). KMT2A rearrangement and the initial FLT3 mutation were still detected with acquisition of a monoallelic complete deletion of IKZF1 (Figure 3B). Salvage treatment consisted of intensive chemotherapy combined with midostaurine BID and triple intrathecal infusions. Bone marrow and extra-medullary CR2 was obtained with undetectable KTM2A breakpoint MRD at level $10^{-5}$. Autologous anti-CD19 CAR-T cells were administered 3 months after the relapse followed by a grade 2 cytokine release syndrome with hematological and neurological toxicities. The child is now 36 months old, in persistent negative MRD CR2 (at mo12 post-CAR $\mathrm{T}$ cells infusion), with no known sequelae to date.

\section{DISCUSSION}

Pediatric MPALs are rare ( $<5 \%$ of pediatric AL cases), with up to $15 \%$ to $20 \%$ failures to achieve CR1. Very few data are available for children with KMT2A-rearranged MPAL (Table 1). Among 28 children treated for a MPAL with lymphoid-type treatment between 1996 and 2006 in the Czech Republic, 3 had a KMT2A/ AFF1 rearrangement, only one being alive after HSCT in CR2 (1). In a more recent series of 39 pediatric MPAL cases treated in Poland from 2007 to 2018, four cases presented with a KMT2A rearrangement but no details are provided regarding their specific treatment and outcome (7). An international cooperative study (18 centers participating in the iBFMAMBI2012 study) looked at the therapeutic strategies and the outcome of 233 children with ambiguous lineage acute leukemia (ALAL) between 2002 and 2015 (176 single-population ALAL, 45 bilineal ALAL, 12 undifferentiated). Fifteen of 233 patients had a KMT2A/AFF1 rearrangement and 11 had another type of $K M T 2 A$ rearrangement. These cases most often aggregate in the group of patients who received a lymphoid and myeloid oriented "hybrid" treatment with a 5y EFS of only $28 \%$ (6).

New concepts and/or therapies and are thus needed for MPAL. BLIN is a commercialized bispecific CD19/CD3 monoclonal antibody. It has been tested for safety and efficacy in children with relapsed/refractory ALL with initial response rates of around $39 \%$. Among 70 patients who received the recommended dosages $\left(15 \mu \mathrm{g} / \mathrm{m}^{2} /\right.$ day after a week at $5 \mu \mathrm{g} / \mathrm{m}^{2} /$ day), the CR rate was $56 \%$ among patients with $<50 \%$ bone marrow blasts at baseline versus $33 \%$ among those with $50 \%$ or more blasts (10). In term or MRD response, among the 27 children who obtained a CR within the first two cycles, 14 (52\%) achieved a complete MRD response, 13 (48\%) by day 15 of cycle 1 (10). This was confirmed in a study comparing BLIN versus historical standard therapy in pediatric $\mathrm{R} / \mathrm{R} \mathrm{Ph}$-negative $\mathrm{B}$-cell precursor ALL (11). In infants less than 2 years of age, the response rate is $60 \%(6 / 10)$ with similar tolerance (10). GO is a commercialized humanized anti-CD33 monoclonal antibody coupled to the cytotoxic antibiotic calicheamicin causing DNA damage after endocytosis. This antibody has been shown to be effective in pediatric AML, including infants with similar and especially reasonable hepatic side effects $(12,13)$. Our main concern was to determine how to associate these two immunotherapies, one targeting the B lymphoid component, the other one targeting the myeloid component of the MPAL. BLIN allows the cytotoxic $\mathrm{T}$ lymphocytes to be engaged with malignant and benignant $\mathrm{B}$ cells, resulting in $\mathrm{B}$ cell lysis and $\mathrm{T}$

TABLE 1 | KMT2A-rerranged MPAL cases from literature and their treatment approach.

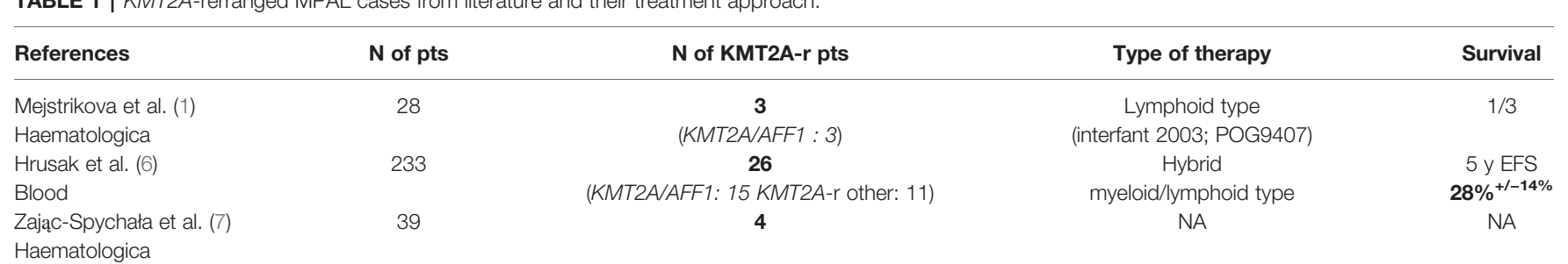


cell expansion. It needs preserved functional effector T-cells. GO, despite being a targeted chemotherapy, could precisely destroy those effectors. This is why we decided to first introduce BLIN alone, to induce the $\mathrm{T}$-cell expansion and action on the $\mathrm{B}$ lineage component of the clone. Then GO was combined from D11 of the onset of BLIN, in a fractionated regimen we previously described as tolerated and effective in children with AML, at a dose of $3 \mathrm{mg} / \mathrm{m} 2$ three times in a week as monotherapy and in combination with cytarabine (14).

The history of our patient highlights the interest of a combined immunotherapy approach tailored to target immunophenotypic markers in MPAL. Indeed, lymphoid and myeloid clones, resistant to conventional chemotherapy, could be controlled by combining two drugs with a curative intent in this patient with a refractory disease. This case report can thus lead to the idea of a sequential combination of two immunotherapies targeting two distinct leukemic subclones (or even a single biphenotypic clone), as a potential one to be tested

\section{REFERENCES}

1. Mejstrikova E, Volejnikova J, Fronkova E, Zdrahalova K, Kalina T, Sterba J, et al. Prognosis of children with mixed phenotype acute leukemia on the basis of consistent immunophenotypic criteria. Haematologica (2010) 95(6):92835. doi: 10.3324/haematol.2009.014506

2. Béné MC. Biphenotypic, bilineal, ambiguous or mixed lineage: strange leukemias! Haematologica (2009) 94(7):891-3. doi: 10.3324/haematol. 2009.007799

3. Vardiman JW, Thiele J, Arber DA, Brunning R, Borowitz M, Porvit A, et al. The 2008 revision of the World Health Organization (WHO) classification of myeloid neoplasms and acute leukemia: rationale and important changes. Blood (2009) 30114(5):937-51. doi: 10.1182/blood-2009-03209262

4. Arber DA, Orazi A, Hasserjian R, Thiele J, Borowitz M, Le Beau M, et al. The 2016 revision to the World Health Organization of myeloid neoplasms and acute leukemia. Blood (2016) 127(20):2391-405. doi: 10.1182/blood-2016-03643544

5. Rubnitz JE, Onciu M, Pounds S, Shurtleff S, Cao X, Raimondi S, et al. Acute mixed lineage leukemia in children: the experience of St Jude Children's Research Hospital. Blood (2009) 113(21):5083-9. doi: 10.1182/blood-200810-187351

6. Hrusak O, de Haas V, Stancikova J, Vakrmanova B, Janotova I, Melstrikova E, et al. International cooperative study identifies treatment strategy in childhood ambiguous lineage leukemia. Blood (2018) 132(3):264-76. doi: 10.1182/blood-2017-12-821363

7. Zając-Spychała O, Irga-Jaworska N, Drożyńska E, Muszynska-Rosla K, Krawczuk-Rybak M, Zawitkowska J, et al. Mixed phenotype acute leukemia: Biological profile, clinical characteristic and treatment outcomes: Report of the population-based study. Eur J Haematol (2020) 105(1):85-93. doi: 10.1111/ejh.13413

8. Pieters R, De Lorenzo P, Ancliffe P, Aversa LA, Brethon B, Biondi A, et al. Outcome of Infants Younger Than 1 Year With Acute Lymphoblastic Leukemia Treated With the Interfant-06 Protocol: Results from an International Phase III Randomized Study. J Clin Oncol (2019) 37 (25):2246-56. doi: 10.1200/JCO.19.00261 prospectively in children MPAL and even possibly all KMT2Arearranged infant ALL.

\section{DATA AVAILABILITY STATEMENT}

The original contributions presented in the study are included in the article/supplementary material. Further inquiries can be directed to the corresponding author.

\section{AUTHOR CONTRIBUTIONS}

$\mathrm{BB}$ reviewed the literature, collected, and analyzed data and wrote the manuscript. $\mathrm{BB}$ and $\mathrm{AB}$ designed the case report. EL, AC-E, and OF provided biological data. All authors contributed to the article and approved the submitted version.

9. Clesham K, Rao V, Bartram J, Ancliff P, Ghorashian S, O'Connor D, et al. Blinatumomab for infant acute lymphoblastic leukemia. Blood (2020) 135 (17):1501-4. doi: 10.1182/blood.2019004008

10. von Stackelberg A, Locatelli F, Zugmaier G, Handgretinger R, Trippett T, Rizzari C, et al. Phase I/phase II study of blinatumomab in pediatric patients with relapsed/refractory acute lymphoblastic leukemia. J Clin Oncol (2016) 34 (36):4381-9. doi: 10.1200/JCO.2016.67.3301

11. Locatelli F, Whitlock JA, Peters C, Chen-Santel C, Chia V, Dennis R, et al. Blinatumomab versus historical standard therapy in pediatric patients with relapsed/refractory $\mathrm{Ph}$-negative $\mathrm{B}$-cell precursor acute lymphoblastic leukemia. Leukemia (2020) 34(9):2473-8. doi: 10.1038/s41375-020-0770-8

12. Gamis AS, Alonzo TA, Meshinchi S, Sung L, Gerbing R, Raimoni S, et al. Gemtuzumab ozogamicin in children and adolescents with de novo acute myeloid leukemia improves event-free survival by reducing relapse risk: results from the randomized phase III Children's Oncology Group trial AAML0531. J Clin Oncol (2014) 32(27):3021-32. doi: 10.1200/JCO.2014.55.3628

13. Guest EM, Aplenc R, Sung L, Raimondi S, Hirsch B, Alonzo T, et al. Gemtuzumab ozogamicin in infants with AML: results from the Children's Oncology Group trials AAML03P1 and AAML0531. Blood (2017) 130 (7):943-5. doi: 10.1182/blood-2017-01-762336

14. Brethon B, Yakouben K, Oudot C, Boutard P, Bruno B, Jerome C, et al. Efficacy of fractionated gemtuzumab ozogamicin with cytarabine in advanced childhood leukaemia. Br J Haematol (2008) 143(4):541-7. doi: 10.1111/j.13652141.2008.07370.x

Conflict of Interest: The authors declare that the research was conducted in the absence of any commercial or financial relationships that could be construed as a potential conflict of interest.

Copyright (C) 2021 Brethon, Lainey, Caye-Eude, Grain, Fenneteau, Yakouben, Roupret-Serzec, Le Mouel, Cavé and Baruchel. This is an open-access article distributed under the terms of the Creative Commons Attribution License (CC BY). The use, distribution or reproduction in other forums is permitted, provided the original author(s) and the copyright owner(s) are credited and that the original publication in this journal is cited, in accordance with accepted academic practice. No use, distribution or reproduction is permitted which does not comply with these terms. 